\title{
THE BROADENING OF METALLIC LINES IN COOL STARS
}

\author{
S. D. ANSTEE, B. J. O'MARA AND J. E. ROSS \\ The University of Queensland, \\ St. Lucia Queensland 4072, Australia
}

\begin{abstract}
A theory for the broadening of spectral lines by collisions with atomic hydrogen developed by Anstee and O'Mara [1] is described and when applied to strong lines of elements in the sun leads to abundances which are independent of non-thermal motions in the photosphere and in excellent agreement with meteoritic values. In the case of the solar abundance of iron the results lead to a resolution of recent conflict concerning possible differences between the abundance of iron in the sun and meteorites. Excellent results obtained with the $\mathrm{Mg}$ b-lines permit these lines to be used in abundance determinations in other stars and also makes them particularly useful in determining the temperature structure in cool stars.
\end{abstract}

\section{Introduction}

In cool stars neutral hydrogen atoms outnumber electrons by four orders of magnitude, consequently the broadening of most spectral lines is dominated by collisions with neutral hydrogen atoms. Conventional van der Waals' theory for this broadening process is known to underestimate the broadening of spectral lines in the sun by about a factor of two. Development of a satisfactory theory is important as it would allow strong lines with well determined $\mathrm{f}$-values to be used to determine abundances in cool stars in a manner which is independent of photospheric motions. Also such lines could also be used to determine surface gravities in cool stars.

\section{Theory Outline}

Collisions with neutral hydrogen atoms are sufficiently fast for the impact approximation of spectral line broadening theory to be valid. In this ap-

T.R. Bedding et al. (eds.),

Fundamental Stellar Properties: The Interaction between Observation and Theory, 179-183.

(C) 1997 IAU. Printed in the Netherlands. 
proximation the line has a Lorentz profile with a half half-width which is given by:

$$
w=N \int_{0}^{\infty} v f(v) \sigma(v) d v
$$

where $N$ is the hydrogen atom number density, $v$ is the relative collision speed, $f(v)$ is the speed distribution and the line broadening cross section

$$
\sigma(v)=2 \pi \int_{0}^{\infty}<\Pi(b, v)>_{a v} b d b
$$

where the integrand contains the product of the geometrical cross section $2 \pi b d b$ and a line broadening efficiency factor $\langle\Pi(b, v)\rangle_{a v}$ for collisions with impact parameter $b$ and relative speed $v$.

$\langle\cdots\rangle_{a v}$ indicates that the efficiency factor has to be averaged over all orientations of the perturbed atom. The efficiency factor can be expressed in terms of the S-matrix elements for the collision which are functionally dependent on the interaction energy between a hydrogen atom in the ground state and the perturbed atom in its upper and lower states. The only essential difference between various theoretical treatments is in the method employed to determine this interaction energy.

In the theory developed by Anstee and O'Mara [1] the interaction energy is calculated using Rayleigh-Schrödinger perturbation theory. If exchange effects are neglected, the shift in energy of the two-atom system as a result of the electrostatic interaction $V$ between them is given by

$$
\Delta E_{i}=<i|V| i>+\sum_{j \neq i} \frac{<i|V| j><j|V| i>}{E_{i}-E_{j}}
$$

where the unperturbed eigenstates of the two-atom system $\mid i>$ are products of the unperturbed eigenstates of the two atoms. As first pointed out by Unsöld the above expression can be greatly simplified if $E_{i}-E_{j}$ can be replaced by a constant value $E_{p}$. Closure can then be used to complete the sum over $\mathrm{j}$ to obtain

$$
\Delta E_{i}=<i|V| i>+\frac{1}{E_{p}}\left(<i\left|V^{2}\right| i>-<i|V| i>^{2}\right)
$$

The second term accounts for the inter-atomic interaction resulting from fluctuations of both atoms simultaneously (dispersion)and the fluctuation of each atom in the static field of the other(induction). The second term dominates the interaction. To develop the theory further the perturbed atom is described by an optical electron outside a positively charged core so that product states of the two-atom system have the form $\left.|100>| n^{*} l m\right\rangle$. 
With reference to the accompanying diagram, the electrostatic interaction energy $V$, in atomic units, is

$$
V=\frac{1}{R}+\frac{1}{r_{12}}-\frac{1}{r_{2}}-\frac{1}{p_{1}}
$$

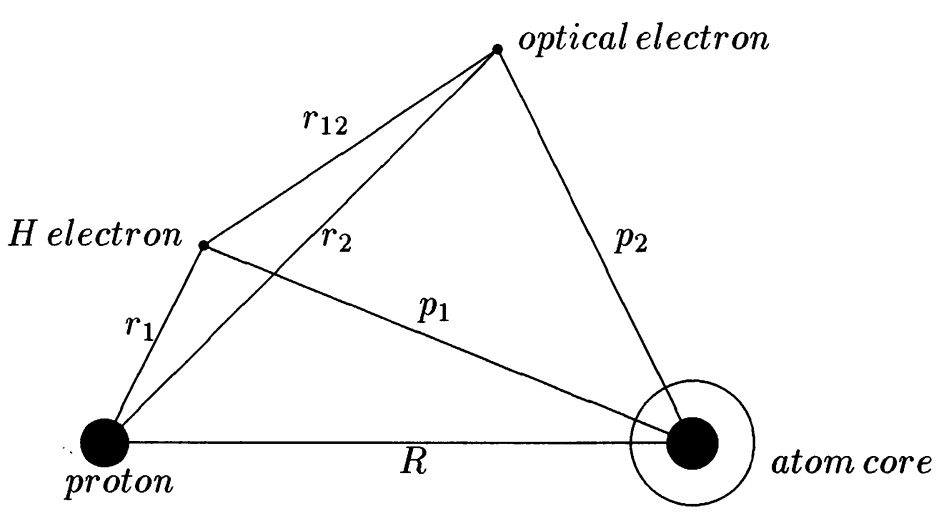

For the state $|i>=| 100>\mid n^{*} l m>$, the interaction energy can be expressed in the form

$$
\begin{aligned}
\Delta E_{n^{*} l|m|}= & <i|V| i>+\frac{1}{E_{p}} \int_{0}^{\infty} R_{n^{*} l}^{2}\left(p_{2}\right) I_{l|m|}\left(p_{2}, R\right) p_{2}^{2} d p_{2} \\
& -\frac{1}{E_{p}}<i|V| i>^{2}
\end{aligned}
$$

where $R_{n^{*} l}\left(p_{2}\right)$ is the radial wave function for the optical electron in the perturbed atom and $I_{l|m|}\left(p_{2}, R\right)$ are lengthy complicated analytic functions of $p_{2}$ and $R$, which have a logarithmic singularity at $p_{2}=R$ and which can be can expressed as an asymptotic expansion in powers of $\frac{1}{R^{2}}$ when $R$ is large. It can be shown that the leading term in this expansion leads to

$$
<\Delta E_{n l|m|}>_{a v} \sim \frac{1}{E_{p}} \frac{<p_{2}^{2}>}{R^{6}}
$$

and if $E_{p}$ is chosen to be $4 / 9$ atomic units $1 / E_{p}=9 / 4$ which is the polarizability of hydrogen in atomic units. Thus

$$
<\Delta E_{n l|m|}>_{a v} \sim \alpha_{H} \frac{<p_{2}^{2}>}{R^{6}}
$$

the standard expression for the van der Waals interaction between the two atoms. However the impact parameters important in the determination of 
the cross section are always too small for this asymptotic form of the interaction to be valid. The terms in $\langle i|V| i\rangle$ can be expressed in a similar but simpler form. It is an important feature of the method that the interaction energy between the two atoms can be determined analytically to within a numerical integration over the radial wave function for the perturbed atom.

For individual transitions of interest Scaled Thomas-Fermi-Dirac or Hartree-Fock radial wave functions can be used in the determination of the interaction energy. Standard methods can then be used to determine the efficiency factor $\langle\Pi(b, v)\rangle_{a v}$ and these can be used to calculate the crosssection and ultimately the line width. This is perhaps the best method for specific lines of interest such as the $\mathrm{Na} \mathrm{D}$-lines and the $\mathrm{Mg}$ b-lines. However without significant loss of accuracy Coulomb wave functions can be used to tabulate cross-sections for a range of effective principal and azimuthal quantum numbers for the upper and lower levels of the transition. This approach enables cross-sections to be obtained for a wide variety of transitions by interpolation. Anstee and O'Mara [2] adopted this approach for s-p and $\mathrm{p}$-s transitions. In addition to tabulating cross-sections for a collisions speed of $v_{0}=10^{4} \mathrm{~ms}^{-1}$ for a range of effective principal quantum numbers for the upper and lower level, they also determined by direct computation, velocity exponents $\alpha$, on the assumption that $\sigma(v) \sim v^{-\alpha}$.

With this dependence of the cross-section on collision speed the integration over the speed distribution can be performed to obtain the line width per unit $\mathrm{H}$-atom density which is given by

$$
\frac{w}{N}=\left(\frac{4}{\pi}\right)^{\alpha / 2} \Gamma\left(\frac{4-\alpha}{2}\right) v_{0} \sigma\left(v_{0}\right)\left(\bar{v} / v_{0}\right)^{1-\alpha},
$$

where $\bar{v}=\left(\frac{8 k T}{\pi \mu}\right)^{1 / 2}$, and $\mu$ is the reduced mass of the two atoms. Typically $\alpha$ is about 0.25 , which leads to a temperature dependence of $T^{0.38}$ for the line width. At present tabulated values of $\sigma$ and $\alpha$ are only available for $\mathrm{s}-\mathrm{p}$ and $\mathrm{p}-\mathrm{s}$ transitions but work is in progress to extend the results to $\mathrm{p}-\mathrm{d}$, $\mathrm{d}-\mathrm{p}$ and $\mathrm{d}-\mathrm{f}$, $\mathrm{f}-\mathrm{d}$ transitions.

\section{Application to the solar spectrum}

The theory has been used by Anstee and O'Mara to synthesize strong lines in the solar spectrum using the spectrum synthesis program of Ross [4] and the solar model of Holweger and Müller [5]. Good fits were obtained to a selection of solar lines using abundances consistent with meteoritic values. More recently the theory has been applied to the solar $\mathrm{Mg}$ b-lines with excellent results. The theory has also been used by Anstee, O'Mara and Ross [3] to determine the solar abundance of iron from strong lines of Fe I. 
The resulting abundance is in agreement in all respects with the meteoritic value, thus ending recent controversy concerning the solar abundance of iron. They also show that the theory accounts both qualitatively and quantitatively for the bifurcation in the empirical curve of growth for iron first observed by Carter [6].

\section{Conclusions}

Strong lines can now be used with some confidence to determine the chemical composition of cool stars, with the derived abundance being independent of non-thermal motions in the atmospheres of these stars. The abundance derived from strong lines can be used to determine the microturbulent velocity from medium-strong lines. Also selected strong lines can be used to obtain surface gravities and to test model atmospheres for cool stars.

\section{References}

1. Anstee S. D. and O'Mara B. J. MNRAS, 253(4A):549, 1991.

2. Anstee S. D. and O'Mara B. J. MNRAS, 276:859, 1995.

3. Anstee S. D., O'Mara B. J., and Ross J. E. MNRAS, 284:202, 1997.

4. Ross J. E. Spectrum synthesis program. http://www.uq.edu.au/ phjross/syn.htm.

5. Holweger H. and Müller E. A. Solar Phys., 39:19, 1974.

6. Carter W. W. Phys. Rev., 76:962, 1949.

\section{DISCUSSION}

ROBERT KURUCZ: What about eight equivalent electrons?

JIM O'MARA: The question refers to the broadening of $\mathrm{FeI}$ lines. I do not believe that there is a problem for lines involving single-electron excitations where the binding energy and hence the effective principal quantum of the optical electron is well defined, as the line-broadening cross-sections are largely determined by the exponential tail of the wave function. There is a definite problem when two-electron excitations are involved. In such cases the binding energy is somewhat ambiguously determined by using the energy of the appropriate excited state of FeII. In future, better line-broadening cross-sections will be calculated for $\mathrm{FeI}$ lines by using more appropriate wave functions. 